\title{
Nondestructive, Automatic and Rapid Determination of Grain Size of Austenitic Stainless Steels by X-ray Diffraction*
}

\section{Introduction}

Mechanical properties are controlled by grain size. Needless to say, it is very important to produce a stainless steel having a certain grain size according to the usage it is intended for. The conventional method of grain size measurement by optical microscopy is not fit to the on line service because it is destructive and time-consuming. The technique for measuring grain size described here is nondestructive, rapid, as well as fully automated, and has actually been applied to the productional annealing and pickling process.

\section{Principle and Procedure of the Technique}

The principle of the technique is schematically shown in Fig. 1. Monochromatic X-ray beam is impinged vertically on the strip of stainless steel and diffracted intensities are measured by counters which are set along the DebyeScherrer diffraction cone of (311) reflecting planes.

Under two assumptions that there is no preferred orientation texture in steel and that the grain size falls within a certain size range, the relation between the grain size (d) and the coefficient of variation (D) of the X-ray intensities ob- tained may be given ${ }^{1)}$ by Eq. (1).

$$
d=k \sqrt{A \cdot r_{l}} \cdot D
$$

where, $k$ is a constant, $A$ is the irradiated area, $r_{l}$ is aperture length along Debye-Scherrer ring, and $D$ is given by Eq. (2)

$$
D=1 / \overline{\mathcal{N}}_{s} \cdot \sqrt{\sum_{i=1}^{n}\left(\mathcal{N}_{s i}-\overline{\mathcal{N}}_{s}\right) / n}
$$

where, $\mathcal{N}_{s i}$ is each of the X-ray intensities obtained, $\overline{\mathcal{N}}_{s}$ is the intensity averaged over all the data, and $n$ is the number of the data.

Although the greater the $n$, the higher will be the reliability of $D$, the available space limits the number of counters to be twenty. To compensate for this shortcoming, the X-ray intensities are measured ten times at different places as the $\mathrm{X}$-ray apparatus is moved through several $\mathrm{mm}$. Two-hundred data are obtained by this process.

As mentioned above, this works on two assumptions. Actually, austenitic stainless steels have a light preferred orientation texture and the structure is sometimes mixed with coarse grains, which can be a cause for error in $D$. However, these effects can be cancelled by the following techniques; 1) The effect of preferred orienta- tion texture on $D$.

For each counter, $D$ is calculated on the data obtained by the ten measurements and then the average value of $D$ is calculated for all the counters.

2) The effect of coarse grains on $D$.

$D$ calculated for all the measured intensities is compared with $D^{*}$ which was calculated excepting the strongest intensity. If the difference between $D$ and $D^{*}$ is within a certain limit, which has been determined experimentally, the $D$ is taken, else $D^{*}$ is taken.

\section{Experimental Results and Actual Operation}

The results obtained on line by this technique is shown in Fig. 2; the grain size obtained by this method is in good agreement with that by optical microscopy. This system is now working on the main annealing and pickling line of our works. The whole system is activated by one button, and the measurement is completed nondestructively and automatically within 1 min.

\section{REFERENCE}

1) K. Hoshino et al.: Tetsu-to-Hagané, 64 (1978), 621.

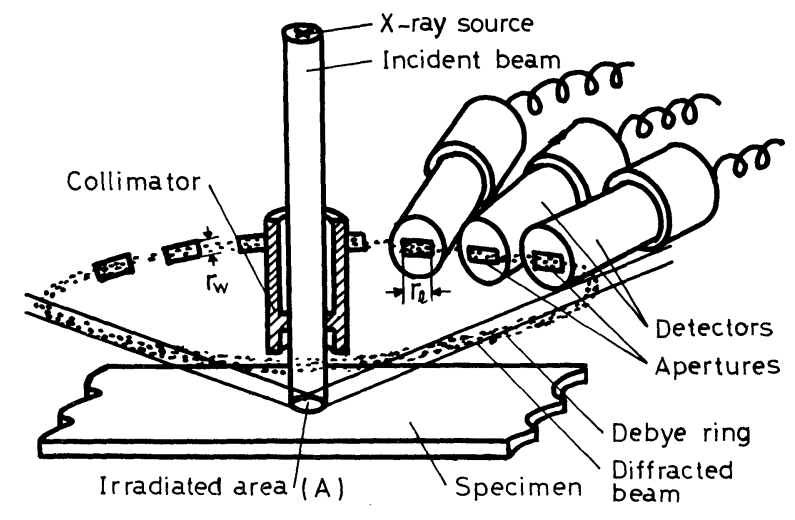

Fig. 1. Schematic diagram of the measuring principle.

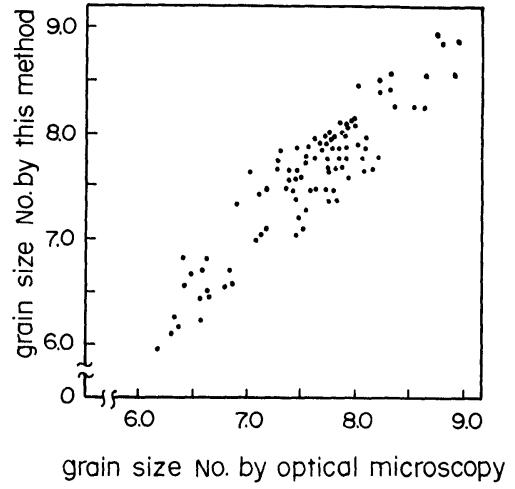

Fig. 2. Comparison of this method with conventional method.

* For further information, write to Shunan Research and Development Laboratories, Nisshin Steel Co., Ltd., 4976, Ōaza Tomita, ShinNanyo 746. (C) 1984 ISIJ 